\title{
TRANSIENT CONTACT INTERACTION OF SOLID BODIES AND THIN SHELLS
}

\author{
DMITRY V. TARLAKOVSKII ${ }^{1}$, GREGORY V. FEDOTENKOV² \\ ${ }^{1}$ Lomonosov Moscow State University, Moscow Aviation Institute \\ 125993, Moscow, Volokolamskoe shosse, 4 \\ tdvhome@mail.ru \\ ${ }^{2}$ Lomonosov Moscow State University, Moscow Aviation Institute \\ 125993, Moscow, Volokolamskoe shosse, 4 \\ greghome@mail.ru
}

Key words: Transient contact problems, Shell, Solid body Superposition method, Fourier series, Integral transforms, Transient functions, Numerical-analytical algorithms.

\begin{abstract}
A spatial transient contact problem with moving boundaries for a thin elastic cylindrical shell and a rigid indenter bounded by a smooth convex surface is considered. A closed mathematical formulation is given and a system of resolving equations is constructed. The main integral equation follows from the principle of superposition and contact conditions. The core of this equation is the transient function for the cylindrical shell. To a closed system of resolving equations, it is supplemented by a kinematic relation for determining the moving boundary of the contact region and the equation of motion of the indenter as a rigid body. An algorithm for solving the spatial non-stationary contact problem for an infinitely long cylindrical shell and rigid indenter in the case of a normal impact on the side surface of the shell is constructed and implemented. Examples of calculations are given.
\end{abstract}

\section{INTRODUCTION}

Transient dynamics of continuous media is one of the most difficult areas of mechanics. The relevance of its study is because, as known, all natural phenomena are nonstationary. The concepts of static and stationary processes are often used as nothing more than an approximation (usually justified) of real phenomena. In many cases, dynamic properties of the medium are necessary, both qualitatively and quantitatively. The study of non-stationary processes is relevant for modern space and aviation technology, shipbuilding, construction of engineering structures, etc.

One has to deal with the issues of unsteady dynamics of elastic shells when solving a wide variety of problems. They find important practical applications in the aerospace, automotive and shipbuilding industries. Their successful solution is associated with the further harmonious development of various sciences: aerohydrodynamics, nonstationary contact problems, acoustics, applied and computational mathematics, and others.

One of the most difficult and least studied problems are transient contact problems with moving boundaries. The complexity of solving problems of this class is due to the mixed and 
nonlinear nature of the boundary conditions, as well as the unknown position of the boundary of the contact area, which moves in time. To solve such problems, approaches based on the method of transient functions (Green's functions) are used [1-7]. Note that these approaches to the solution are based on a preliminary analytical solution of problems on the construction of influence functions and are a convenient tool for solving problems of nonstationary dynamics of elastic bodies, including nonstationary inverse problems. Approaches based on the finite element method and other numerical methods are also possible.

In this paper, we propose a method and an algorithm for solving transient contact problems for cylindrical shell and rigid indenter. It is based on the application of the method of transient functions, according to which, first, by analytical methods, a fundamental solution (transient function) for a shell is constructed $[1,7]$. Then, based on the principle of superposition, the problem is reduced to a system of resolving equations. The resulting system is solved using a numerical-analytical algorithm based on the grid method. Note that, despite the large number of works devoted to the mechanics of shells and contact problems, there are practically no publications devoted to solving spatial transient contact problems. The constructed technique can serve as a basis for the development of mathematical formulations and methods for solving new transient contact problems as well as for contact problems taking into account anisotropic combined dry friction [8-12].

\section{STATEMENT OF THE PROBLEM}
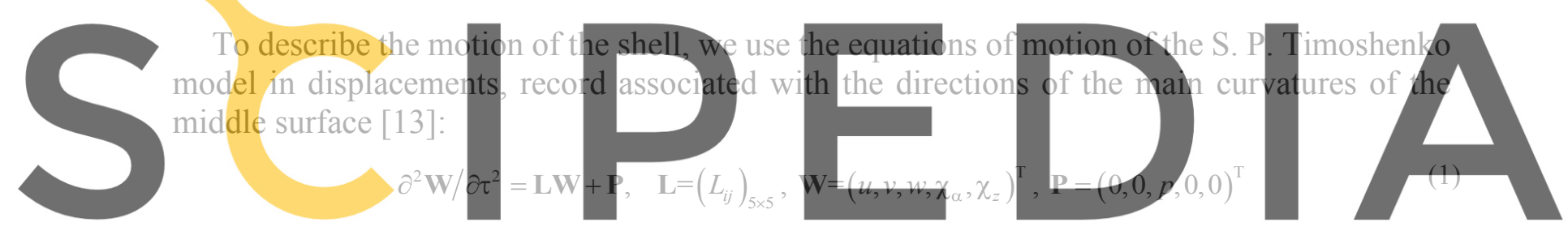

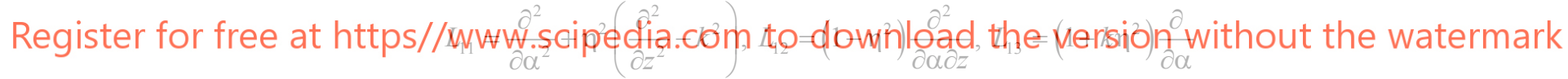

$$
\begin{gathered}
L_{14}=-\gamma^{2} \frac{\partial^{2}}{\partial \alpha^{2}}+\eta^{2} k^{2}, L_{15}=-\gamma^{2}\left(1-2 \eta^{2}\right) \frac{\partial^{2}}{\partial \alpha \partial z}, L_{21}=L_{12}, L_{22}=\frac{\partial^{2}}{\partial z^{2}}+\eta^{2} \frac{\partial^{2}}{\partial \alpha^{2}} \\
L_{23}=\left(1-2 \eta^{2}\right) \frac{\partial}{\partial z}, L_{24}=-\gamma^{2} \eta^{2} \frac{\partial^{2}}{\partial \alpha \partial z}, L_{25}=-\gamma^{2} \eta^{2} \frac{\partial^{2}}{\partial \alpha^{2}}, L_{31}=-L_{13} \\
L_{32}=-L_{23}, L_{33}=\eta^{2} k^{2}\left(\frac{\partial^{2}}{\partial \alpha^{2}}+\frac{\partial^{2}}{\partial z^{2}}\right)-1, L_{34}=\eta^{2} k^{2} \frac{\partial}{\partial \alpha}, L_{35}=\eta^{2} k^{2} \frac{\partial}{\partial z} \\
L_{41}=\gamma^{-2} L_{14}, L_{42}=\gamma^{-2} L_{24}, L_{43}=-\gamma^{-2} L_{34}, L_{44}=\frac{\partial^{2}}{\partial \alpha^{2}}+\eta^{2}\left(\frac{\partial^{2}}{\partial z^{2}}-k^{2} \gamma^{-2}\right) \\
L_{45}=L_{12}, L_{51}=\gamma^{-2} L_{15}, L_{52}=\gamma^{-2} L_{25}, L_{53}=-\gamma^{-2} L_{35} \\
L_{54}=L_{12}, L_{55}=\frac{\partial^{2}}{\partial z^{2}}+\eta^{2}\left(\frac{\partial^{2}}{\partial \alpha^{2}}-k^{2} \gamma^{-2}\right)
\end{gathered}
$$

$u, v$-tangential displacements, $w$ - normal displacement, $\chi_{\alpha}, \chi_{z}$ - normal fiber rotation angles, $\mathbf{P}$ - external forces, $\tau$-dimensionless time, $\mathbf{L}$ is the matrix-operator. All variables and parameters are reduced hereinafter to a dimensionless form as follows (primed variables 
correspond to dimensional ones):

$$
u=R^{-1} u^{\prime}, v=R^{-1} v^{\prime}, w=R^{-1} w^{\prime}, u_{c}=R^{-1} u_{c}{ }^{\prime}, z=R^{-1} z^{\prime}, \tau=R^{-1} c_{1} t, \eta^{2}=c_{2}^{2} c_{1}^{-2}, c_{1}^{2}=\rho^{-1}(\lambda+2 \mu)
$$

$\rho$ - mass density, $\lambda, \mu$ - Lame parameters, $R$ and $h$ - radius and thickness of the shell, $u_{c}$ - displacement of the center of mass of the indenter, $c_{1}$ and $c_{2}-$ speed of the tensioncompression waves and shear waves in the shell material, $t$-dimensional time.

At the initial moment of time, an absolutely solid impactor, moving with a given initial velocity, comes into contact with the lateral surface of the infinitely long thin elastic circular cylindrical shell. The impactor is a solid body with a smooth convex surface, the vector of its initial velocity is directed normal to the side surface of the shell (Fig. 1).

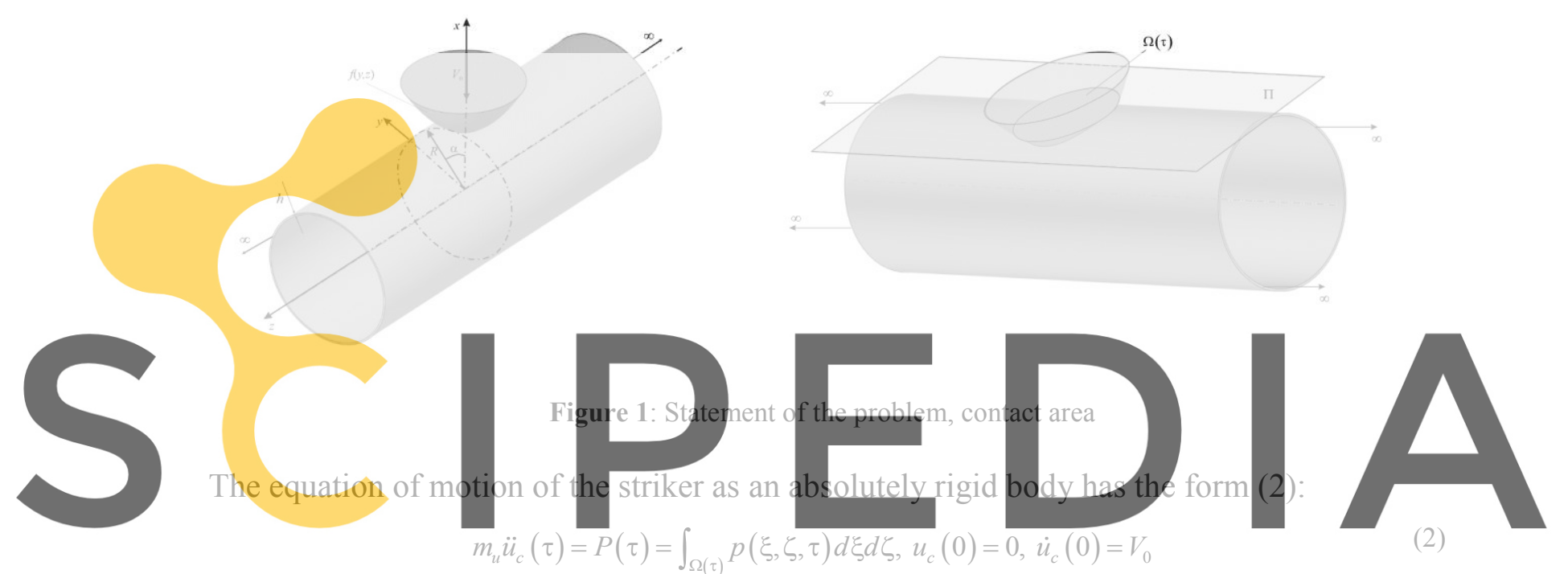

Register for free at https//www.scipedia.com to download the version without the watermark

\section{depends on time.}

We assume that the contact between the shell and the striker occurs under conditions of free sliding. Let $\Pi_{1}$ and $\Pi_{2}$ be the surfaces bounding the shell and the striker at the moment of time $\tau>0$. With respect to an inertial Cartesian rectangular frame whose axis coincides with the axis of the shell, they are given by the equations

$$
\Pi_{k}: x=f_{k}(y, z, \tau),(y, z) \in \Omega_{k}, k=1,2
$$

$\Omega_{k}$ - projections of surfaces $\Pi_{1}$ and $\Pi_{2}$ on a plane $O y z$. The true contact area is defined by the conditions (3):

$$
\Pi_{*}: \Delta(y, z, \tau)=f_{1}(y, z, \tau)-f_{2}(y, z, \tau)=0, p(y, z, \tau)<0,(y, z) \in \Omega_{k}
$$

where $\Delta-$ is the gap between the boundary surfaces $\Pi_{1}$ and $\Pi_{2}$. The equations that implicitly define the boundary $\partial \Pi_{*}$ of the contact area follow from (3):

$$
\partial \Pi_{*}: \Delta(y, z, \tau)=0, p(y, z, \tau)=0
$$


In the linearized formulation, the true contact area $\Pi_{*}$ is replaced by a fictitious area $\Omega(\tau)$ belonging to the plane that is a common tangent to the surfaces $\Pi_{1}, \Pi_{2}$ at the initial time of interaction (Fig. 1). Suppose that the position of the surface bounding the striker at the time $\tau$ is given by the equation referred to the frame Oxyz:

$$
x=f(y, z)+u_{c}(\tau)
$$

The boundary $\partial \Omega(\tau)$ of the area $\Omega(\tau)$ is determined from the condition of intersection of the surface bounding the striker with the plane $\Pi$ :

$$
\partial \Omega(\tau): f(y, z)+u_{c}(\tau)=1
$$

Taking into account (4), the contact conditions of the indenter and the shell have the form

$$
f(y, z)+u_{c}(\tau)=w(\alpha, z, \tau), p(\alpha, z, \tau)<0, y, z \in \Omega(\tau), y=\sin \alpha
$$

At the initial time, the shell is in an unreformed state, which corresponds to zero initial conditions

$$
\left.u\right|_{\tau=0}=\left.v\right|_{\tau=0}=\left.w\right|_{\tau=0}=\left.\chi_{\alpha}\right|_{\tau=0}=\left.\chi_{z}\right|_{\tau=0}=\left.\dot{u}\right|_{\tau=0}=\left.\dot{v}\right|_{\tau=0}=\left.\dot{w}\right|_{\tau=0}=\left.\dot{\chi}_{\alpha}\right|_{\tau=0}=\left.\dot{\chi}_{z}\right|_{\tau=0}=0
$$

Equations and relations (1), (2), (4)-(7) are a closed mathematical formulation of the nonstationary contact problem with movable boundaries for a cylindrical shell and a rigid indenter.
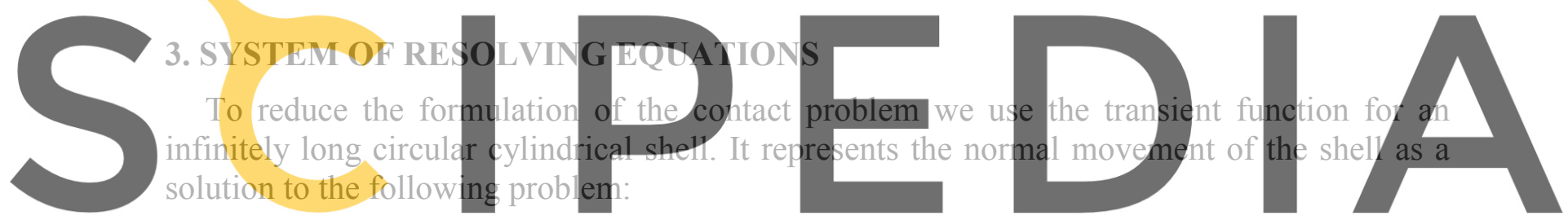

$$
\partial^{2} \mathbf{G} / \partial \tau^{2}=\mathbb{L G}+\mathbf{Q}, \mathbb{L}=\left(L_{i i}\right)_{5 \times 5}, \mathbf{G}=\left(G_{u}, G_{v}, G_{w}, G_{\chi 1}, G_{x 2}\right)^{\mathrm{T}}, \mathbf{Q}=[0,0, \delta(\alpha) \delta(z) \delta(\tau), 0,0]^{\mathrm{T}}
$$

Register for free at https//www.scipedia.com to download the version without the watermark

$\delta(\alpha), \delta(z), \delta(\tau)$ are the Dirac functions. The transient function was found in [7].

Based on the superposition principle [1-7], deflections are related to contact pressures by means of the convolution

$$
w(\alpha, z, \tau)=\int_{0}^{\tau} d t \iint_{\Pi_{*}(t)} G_{w}(\alpha-\xi, z-\zeta, \tau-t) p(\xi, \zeta, t) d S
$$

Replace the contact area $\Pi_{*}(\tau)$ with a dummy area $\Omega(\tau)$. Since $\operatorname{diam} \Omega(\tau)<<1$, we assume

$$
\sin \alpha \approx \alpha \approx y,(y, z) \in \Omega(\tau)
$$

From contact condition (6), (8) and (9), the main integral equation for the sought contact pressure is implies

$$
\int_{0}^{\tau} d t \iint_{\Omega(t)} G_{w}(y-\xi, z-\zeta, \tau-t) p(\xi, \zeta, t) d S=f(y, z)+u_{c}(\tau)
$$

In equation (10), in addition to the contact pressure, the contact area $\Omega(\tau)$ and the 
displacement of the center of mass of the indenter $u_{c}(\tau)$ are also unknown. To obtain a closed system, we add (5) which determines the position of the boundary of the contact region, and the equation of motion of the striker (2), written in the integral form

$$
u_{c}(\tau)=V_{0} \tau+\frac{1}{m_{u}} \int_{0}^{\tau}(\tau-t) d t \iint_{\Omega(t)} p(y, z, t) d S
$$

\section{SOLUTION METHOD}

To solve the system of resolving equations, we use a numerical-analytical algorithm based on the method of mechanical quadrature. We divide the time interval $[0, \tau]$ into $M$ uniform steps $\Delta_{t}=\tau / M: t_{m}=m \Delta_{t}, m=\overline{1, M}, t_{M}=M \Delta_{t}=\tau$. We replace the time integrals in (10) and (11) by the sum of the integrals over the intervals $\left[\tau_{m-1}, \tau_{m}\right]$ and assume that the contact pressure remains constant within each time interval:

$$
\begin{gathered}
\sum_{m=1}^{M} \int_{t_{m-1}}^{t_{m}} d t \iint_{\Omega(t)} G_{w}(y-\xi, z-\zeta, \tau-t) p_{m}(\xi, \zeta) d S=f(y, z)+u_{c M} \\
u_{c M}=V_{0} \tau+\frac{1}{m_{u}} \sum_{m=1}^{M} \int_{\tau_{m-1}}(\tau-t) d t \iint_{\Omega(t)} p_{m}(\xi, \zeta) d S, u_{c M}=u_{c}(\tau), p_{m}(\xi, \zeta)=p\left(\xi, \zeta, t_{m}\right), t_{m}=m \Delta_{t}
\end{gathered}
$$
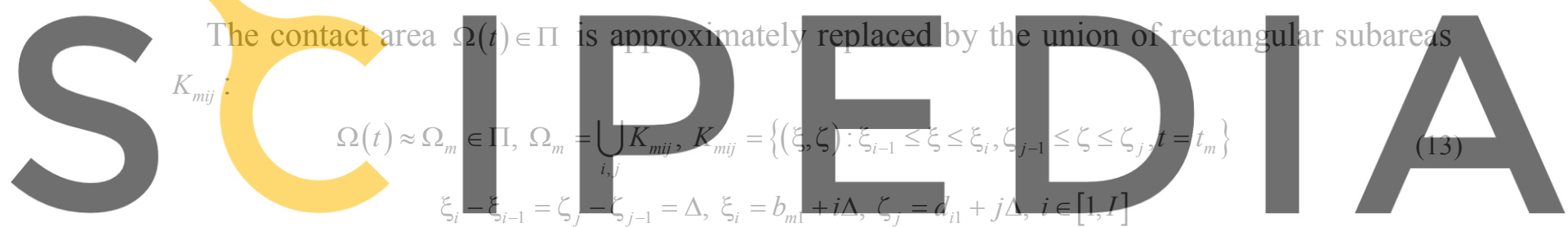

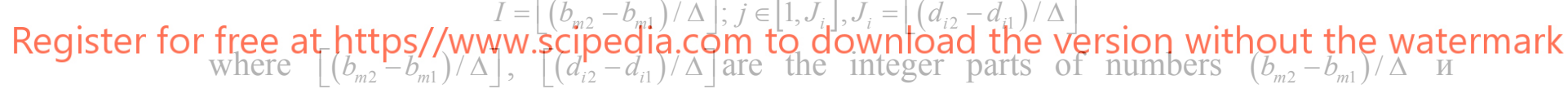

$\left(d_{i 2}-d_{i 1}\right) / \Delta$.

To secure the stability of the numerical scheme, the step $\Delta$ in the spatial variables is selected from the condition $\Delta>\Delta_{t}$ [6]. In particular, we can put $\Delta=2 \Delta_{t}$. The boundaries $b_{m 1}, b_{m 2}, d_{i 1}, d_{i 2}$ changes in discrete values of spatial variables $\xi_{i}$ and $\zeta_{j}$ are solutions of the equations

$$
f\left(b_{m}, 0\right)+u_{c m}=1 \Rightarrow b_{m 1}, b_{m 2}, f\left(\xi_{i}, d_{i}\right)+u_{c m}=1 \Rightarrow d_{i 1}, b_{i 2}, u_{c m}=u\left(t_{m}\right)
$$

Using (13), we obtain numerical analogs of equations (12). To do this, we assume that the contact pressure remains constant within an elementary square $K_{m i j}:\left.p_{m}(\xi, \zeta)\right|_{(\xi, \zeta) \in K_{i j}}=p_{m i j}=$ const . Then from (12) - (14) follows 


$$
\begin{gathered}
\sum_{m=1}^{M} \sum_{i=1}^{I} \sum_{j=1}^{J_{i}} G_{m i j k l} p_{m i j}=f_{k l}+u_{c M}, u_{c M}=V_{0} \tau+\frac{\Delta_{t}^{2} \Delta^{2}}{m_{u}} \sum_{m=1}^{M} A_{m} \sum_{i=1}^{I} \sum_{j=1}^{J_{i}} p_{m i j}, f\left(b_{m}, 0\right)+u_{c m}=1 \Rightarrow \\
b_{m 1}, b_{m 2}, f\left(\xi_{i}, d_{i}\right)+u_{c m}=1 \Rightarrow d_{i 1}, d_{i 2}, k, i \in[1, I], I=\left[\left(b_{m 2}-b_{m 1}\right) / \Delta\right] \\
l, j \in\left[1, J_{i}\right], J_{i}=\left[\left(d_{i 2}-d_{i 1}\right) / \Delta\right] \\
G_{m i j k l}=\int_{t_{m-1}}^{t_{m}} \int_{\xi_{i-i}}^{\xi_{i}} \int_{\zeta_{j-i}}^{\zeta_{j}} G_{w}\left(y_{k}-\xi, z_{l}-\zeta, M \Delta_{t}-t\right) d \xi d \zeta d t, A_{m}=M-m+\frac{1}{2}
\end{gathered}
$$

\section{NUMERICAL EXAMPLES}

As an example, consider the unsteady contact interaction of a circular cylindrical shell, of radius $R=1$ and thickness $h=0.025$, with an absolutely rigid indenter bounded by a surface in the form of a paraboloid of revolution: $f(y, z)=y^{2}+z^{2}+1$. As a shell material, we take steel with the following characteristics: $\rho=7850 \mathrm{~kg} / \mathrm{m}^{3}, \lambda=1.15 \times 10^{11} \mathrm{~Pa}, \mu=7.69 \times 10^{10} \mathrm{~Pa}$, which is advised by dimensionless parameters: $\eta=0.53$ ,$\gamma=1.3 \times 10^{-6}$

Let us consider the influence of the initial velocity of the striker on the process of unsteady contact interaction. Let's take three of its possible values for analysis $V_{0}=0.01, V_{0}=0.05$ and $V_{0}=0.1$. The contact surface in this case is a circle whose boundary coincides with the section of the surface $x=y^{2}+z^{2}+1-u_{c}(\tau)$ by a plane $x=1$ at the current time.

Fig. 2a-2c shows the time dependence of the displacement of the center of mass of the striker (Fig. 2a), the radius of the contact area on time (Fig, $2 \mathrm{~b}$ ) and the resulting contact force $P(\tau)$ versus time (Fig. 2c). Here the

solid curves correspond to

Fig. 3a-3c shows the distr

Fig. 3 a corresponds to the c

dashed $-V_{0}=0.05$, dash-do

Fig. $4 \mathrm{a}-4 \mathrm{c}$ shows the distribution of the contact press
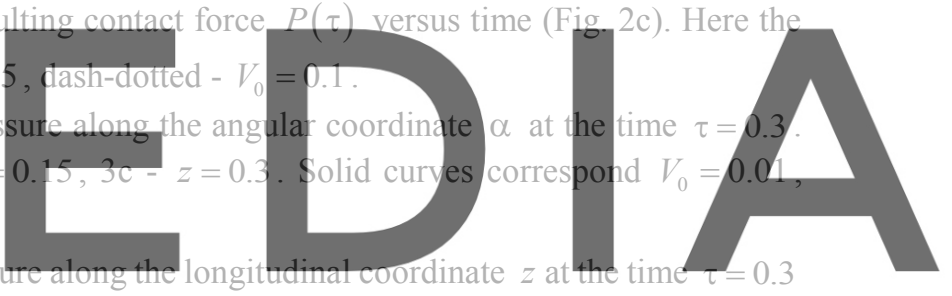

Fig. 4 a corresponds to the value of the coordinate $\alpha=0,4 b-\alpha=0.15,4 c-\alpha=0.3$. Solid curves correspond

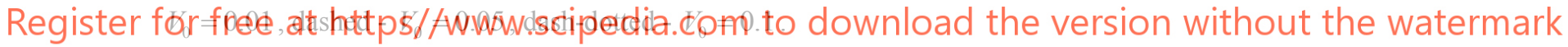

Fig. $5 \mathrm{a}$ and $5 \mathrm{~b}$ shows the distributions of normal displacements along the angular coordinate (Fig. 5a) and along the longitudinal coordinate (Fig. 5b). Here, the results correspond to the point in time $\tau=0.6$ and the initial speed $V_{0}=0.1$. In fig. 5a, solid curves correspond $z=0$, dashed $-z=0.15$, dash-dotted $-z=0.3$. Solid curves in Fig. $5 b$ correspond $\alpha=0$, dashed $-\alpha=0.15$, dash-dotted $-\alpha=0.3$.

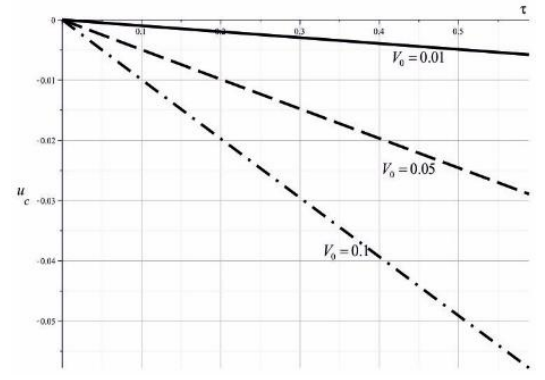

(a)

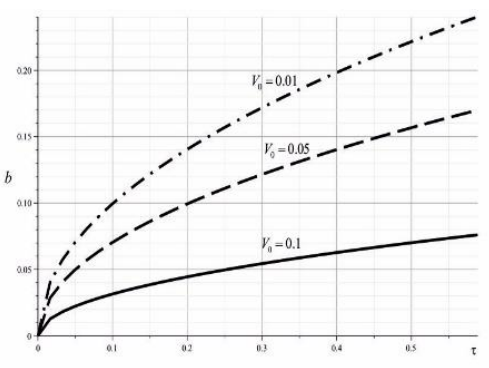

(b)

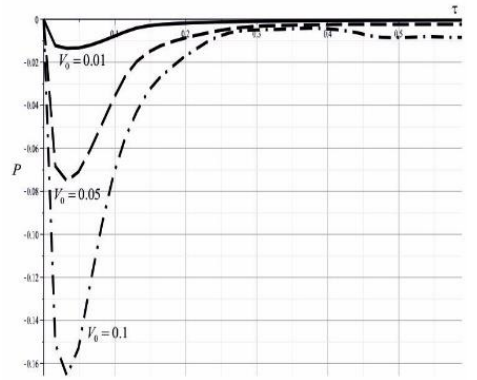

(c)

Figure 2: Dependences of the displacement of the center of mass of the striker, the radius of the contact area and the resulting contact force on time. 


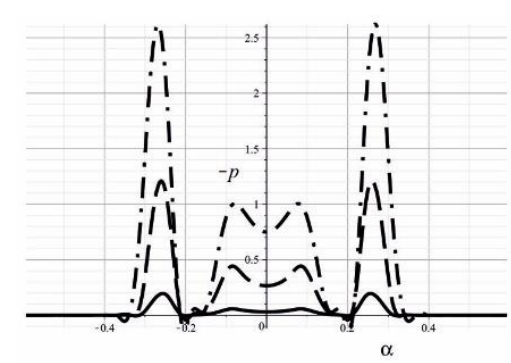

(a)

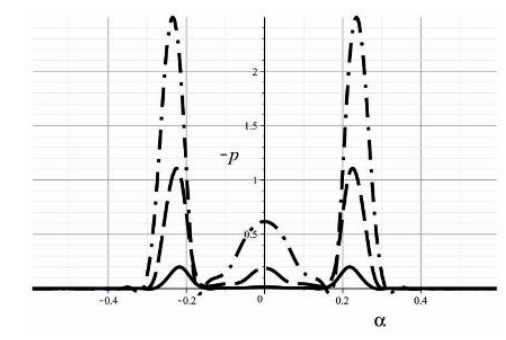

(b)

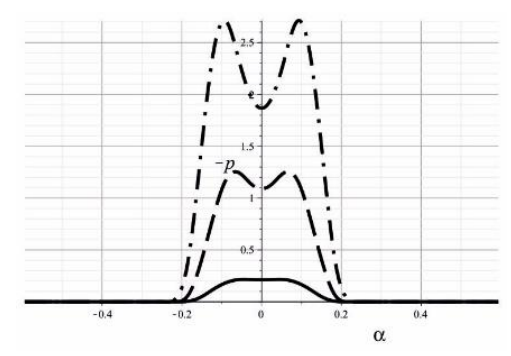

(c)

Figure 3: Distribution of contact pressure along the angular coordinate at $\tau=0.3$.

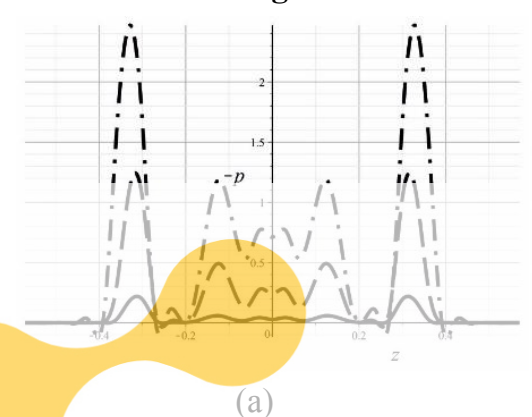

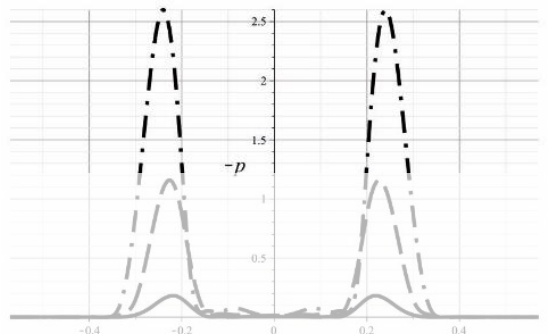

(b)

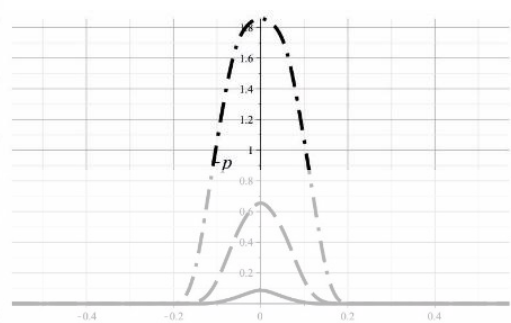

(c)

Figure 4: Distribution of contact pressure along the longitudinal coordinate at $\tau=0.3$
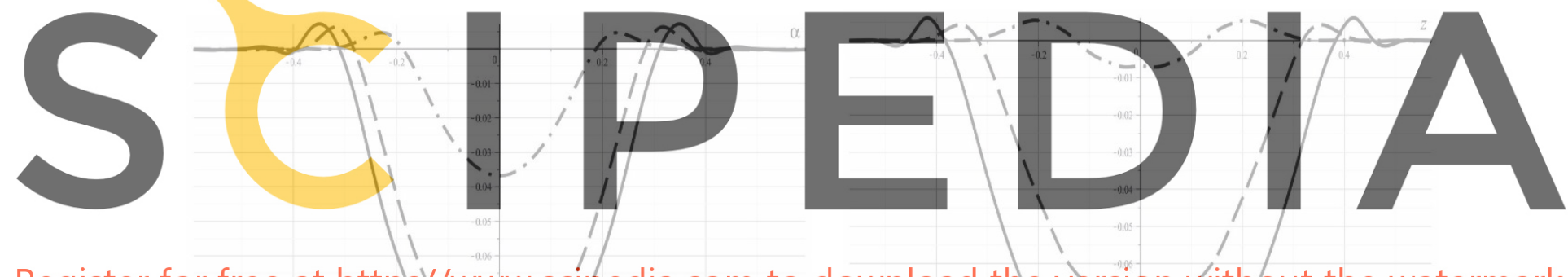

Register for free at httpsy/www.scipedia.com to download the version wwithout the watermark

(a)

(b)

Figure 5: Distribution of normal displacements along the angular and longitudinal coordinates.

\section{ACKNOWLEDGMENTS}

This work was supported financially by the Russian Foundation for Basic Research (projects 20-08-01120, 20 58-00023, 19-08-00438)

\section{REFERENCES}

[1] Fedotenkov, G. and Tarlakovskii, D. Non-stationary contact problems for thin shells and solids. Structural Integrity (2020) 16: 287-292. DOI: 10.1007/978-3-030-47883-4_51

[2] Vestyak, A.V., Igumnov, L.A., Tarlakovskii, D.V. and Fedotenkov, G.V. The influence of non-stationary pressure on a thin spherical shell with an elastic filler. // Computational Continuum Mechanics (2016) 9(4): 443-452. (DOI: 10.7242/1999-6691/2016.9.4.37).

[3] Mikhailova, E.Y., Tarlakovski,i D.V. and Fedotenkov, G.V. Transient contact problem for 
liquid filled concentric spherical shells and a rigid barrier. Structural Integrity (2019) 5: 389-391. (DOI: 10.1007/978-3-319-91989-8_92).

[4] Fedotenkov, G.V., Mikhailova, E.Yu., Kuznetsova, E.L. and Rabinskiy, L.N. Modeling the unsteady contact of spherical shell made with applying the additive technologies with the perfectly rigid stamp. International Journal of Pure and Applied Mathematics (2016) 111 (2): 331-342. (DOI: 10.12732/ijpam.v111i2.16).

[5] Tarlakovskii, D.V. and Fedotenkov, G.V. Nonstationary 3D motion of an elastic spherical shell. Mechanics of Solids (2015) 50(2): 208-217. DOI: 10.3103/S0025654415020107

[6] Mikhailova, E.Y. and Fedotenkov, G.V. Nonstationary axisymmetric problem of the impact of a spherical shell on an elastic half-space (initial stage of interaction). Mechanics of Solids (2011) 46(2): 239-247. (DOI: 10.3103/S0025654411020129).

[7] Fedotenkov, G.V. Kalinchuk, V.V. and Mitin A.Y. Lobachevskii J. of Mathematics (2019) 40(3): 311-320. (DOI: 10.1134/S1995080219030107).

[8] Kireenkov A.A. and Zhavoronok S.I., Anisotropic Combined Dry Friction in Problems of Pneumatics' Dynamics. Journal of Vibrational Engineering and Technologies (2020), 8(2): 365-372. (DOI: 10.1007/s42417-019-00140-1).

[9] Kireenkov, A.A. Improved friction model of the aviation tyre contact with the landing strip. IFAC-PapersOnLine (2018) 51(2): 890-894. (DOI 10.1016/j.ifacol.2018.04.027).

[10] Kireenkov, A.A. and Zhavoronok, S.I. Implementation of analytical models of the anisotropic combined dry friction in problems of pneumatics' dynamics. MATEC Web of Conferences (2018) 211(08004). (DOI 10.1051/matecconf/201821108004).
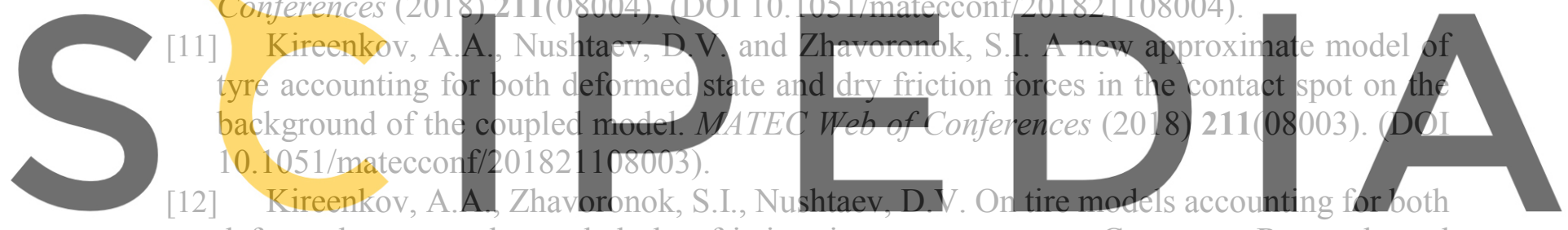

deformed state and coupled dry friction in a contact spot. Computer Research and

Register for freed eatn

[13] Mihajlova, E.Y., Tarlakovskii, D.V. and Fedotenkov, G.V. A generalized linear model of dynamics of thin elastic shells. Uchenye Zapiski Kazanskogo Universiteta. Seriya FizikoMatematicheskie Nauki (2018) 160(3): 561-577. (2-s2.0-85087810884). 